IJMMS 29:5 (2002) 291-301

PII. S0161171202005124

http://ijmms.hindawi.com

(c) Hindawi Publishing Corp.

\title{
ON UNIFIED THEORY FOR CONTINUITIES
}

\section{MAHIDE KÜÇÜK and YALÇIN KÜÇÜK}

Received 3 March 2000

\begin{abstract}
The purpose of this study is to give a unified theory for some weak and strong continuous
\end{abstract} functions using operations.

2000 Mathematics Subject Classification: 54C10, 54D20, 54D30.

1. Introduction. It is well known that a large number of papers is devoted to the study of weak continuity and strong continuity definitions between topological spaces. Much of these were given using open, semiopen, preopen concepts, and so forth in topological spaces. Their theories run, either in part or in whole, parallel to their theory of continuous functions. Some authors like Kohli [8] and Kandil [7] tried to unify their definitions and properties.

Throughout the present paper, $(X, \tau)$ means topological space on which no separation axioms are assumed unless otherwise explicitly stated.

Let $A$ be a subset of $X$, the closure, semiclosure, and interior of $A$ will be denoted by $\operatorname{cl} A, \operatorname{scl} A$, and $\operatorname{int} A$, respectively. The subset $A$ is called semiopen [10] (resp., preopen [12], $\alpha$-open [15], and $\beta$-open [2]) if $A \subset \operatorname{cl}(\operatorname{int} A)$ (resp., $A \subset \operatorname{int}(\operatorname{cl} A), A \subset$ $\operatorname{scl}(\operatorname{int} A)$, and $A \subset \operatorname{cl}(\operatorname{int}(\operatorname{cl} A)))$.

DefinITION 1.1 [7]. Let $(X, \tau)$ be a topological space. A mapping $\varphi: P(X) \rightarrow P(X)$ is called an operation on $P(X)$, where $P(X)$ denotes the family of all the subsets of $X$, if and only if for each $A \in P(X)-\{\phi\}$, int $A \subset A^{\varphi}$ and $\phi=\phi^{\varphi}$, where $\mathrm{A}^{\varphi}$ denotes the value of $\varphi$ in $A$. The class of all operations on $P(X)$ is denoted by $O_{(X, \tau)}$.

DEFINITION 1.2 [7]. Let $(X, \tau)$ be a topological space. A partial order " $\leq$ " on $O_{(X, \tau)}$ is defined in the following way: $\varphi_{1} \leq \varphi_{2} \Leftrightarrow A^{\varphi_{1}} \subseteq A^{\varphi_{2}}$, for each $A \subseteq X$, where $\varphi_{1}, \varphi_{2} \in$ $O_{(X, \tau)}$.

DEFINITION 1.3 [7]. An operation $\varphi \in O_{(X, \tau)}$ is called monotonous if and only if $A \subseteq B \Rightarrow A^{\varphi} \subseteq B^{\varphi}$, for each $A, B \subseteq X$.

Throughout, all the operations on $P(X)$ are assumed to be monotonous.

Definition 1.4. Let $(X, \tau)$ be a topological space, $G, H \in P(X)$, and $\varphi \in O_{(X, \tau)}$. Then

(i) $G$ is called $\varphi$-open if and only if $G \subseteq G^{\varphi}$,

(ii) $H$ is called $\varphi$-closed if and only if $X-H$ is $\varphi$-open.

- If $X^{\varphi}=X$ then each set of the form $G^{\varphi}$ is $\varphi$-open.

- If each $G \in P(X), G \subseteq G^{\varphi}$, then all the subsets of $X$ are $\varphi$-open. 
- If each $G \in P(X), G \subseteq G^{\varphi}$, then $G$ is $\varphi$-open if and only if $G=G^{\varphi}$.

- If we take $\varphi=$ int, then $\varphi$-open sets are open sets.

- Any union of $\varphi$-open sets is $\varphi$-open [5].

The class of all $\varphi$-open ( $\varphi$-closed) subsets of $X$ is denoted by $O_{\varphi}(X)\left(C_{\varphi}(X)\right)$. For each $x \in X$, the class of $\varphi$-open neighborhoods of $x$ is denoted by $N_{\varphi}(X, x)$, that is, $N_{\varphi}(X, x)=\left\{V \subseteq X: x \in V \in O_{\varphi}(X)\right\}$.

The definitions of $\varphi_{1,2}$-closure and $\varphi_{1,2}$-interior of any subset $A$ of $X$ were given by El-Monsef et al. [1]. Similarly, the definition $\varphi_{1,2}$-frontier of $A$ can be given in the following way.

Definition 1.5. Let $(X, \tau)$ be a topological space, $x \in X, A \in P(X)$, and $\varphi_{1}, \varphi_{2} \in$ $O_{(X, \tau)}$. Then

(i) the $\varphi_{1,2}$-closure of $A$, denoted by $\varphi_{1,2}$ - $\operatorname{cl} A$, is defined by $x \in \varphi_{1,2}$ - $\operatorname{cl} A \Leftrightarrow A \cap$ $U^{\varphi_{2}} \neq \varnothing$, for all $U \in N_{\varphi_{1}}(X, x)$ (see [7]);

(ii) the $\varphi_{1,2}$-interior of $A$, denoted by $\varphi_{1,2}$-int $A$, is defined by $x \in \varphi_{1,2}$-int $A \Leftrightarrow$ $U^{\varphi_{2}} \subseteq A$, for some $U \in N_{\varphi_{1}}(X, x)$ (see [7]);

(iii) the $\varphi_{1,2}$-frontier of $A$, denoted by $\varphi_{1,2}-\partial A$, is defined by $x \in \varphi_{1,2}-\partial A \Leftrightarrow A \cap$ $U^{\varphi_{2}} \neq \varnothing$ and $(X-A) \cap U^{\varphi_{2}} \neq \varnothing$, for all $U \in N_{\varphi_{1}}(X, x)$.

DeFinITION 1.6. Let $(X, \tau)$ be a topological space, $A \in P(X)$ and $\varphi_{1}, \varphi_{2} \in O_{(X, \tau)}$. Then

(i) $A$ is $\varphi_{1,2}$-open if and only if $A \subset \varphi_{1,2}$-int $A$.

(ii) $A$ is $\varphi_{1,2}$-closed if and only if $\varphi_{1,2^{-}} \mathrm{cl} A \subset A$.

The class of all $\varphi_{1,2}$-open ( $\varphi_{1,2}$-closed) subsets of $X$ is denoted by $O_{\varphi_{1,2}}(X)$ $\left(C_{\varphi_{1,2}}(X)\right)$. For each $x \in X$, the class of $\varphi_{1,2}$-open neighborhoods of $x$ is denoted by $N_{\varphi_{1,2}}(X, x)$, that is, $N_{\varphi_{1,2}}(X, x)=\left\{V \subseteq X: x \in V \in O_{\varphi_{1,2}}(X)\right\}$ (see [7]).

Proposition 1.7. Let $(X, \tau)$ be a topological space and $A \subseteq X$. Then the following statements are true:

(1) $\varphi_{1,2}-\partial A=\varphi_{1,2}-\mathrm{cl} A \cap \varphi_{1,2}-\operatorname{cl}(X \backslash A)$.

(2) $\varphi_{1,2}-\partial A=\varphi_{1,2}-\operatorname{cl} A-\left(\varphi_{1,2}-\operatorname{int} A\right)$.

(3) $\varphi_{1,2}-\mathrm{cl} A=\varphi_{1,2}-\partial A \cup \varphi_{1,2}$-int $A$.

Proof. The proof is immediate by definition of $\varphi_{1,2}$-frontier.

2. Unified framework. The definition of $\varphi_{1,2} \psi_{1,2}$-continuity of any function $f$ defined from a topological space $(X, \tau)$ to a topological space $(Y, \vartheta)$ can be given in the following way.

Definition 2.1. Let $(X, \tau),(Y, \vartheta)$ be topological spaces. A function $f:(X, \tau) \rightarrow$ $(Y, 9)$ is said to be $\varphi_{1,2} \psi_{1,2}$-continuous at a point $x \in X$ if for each $V \in O_{\psi_{1}}(Y)$ with $f(x) \subseteq V$, there exists $U \in O_{\varphi_{1}}(X, x)$ such that $f\left(U^{\varphi_{2}}\right) \subseteq V^{\psi_{2}}$ or $U^{\varphi_{2}} \subseteq f^{-1}\left(V^{\psi_{2}}\right)$. If $f$ has this property at each point $x \in X$, then $f$ is $\varphi_{1,2} \psi_{1,2}$-continuous on $X$.

The above definition plays a very important unification role in topology, because $\varphi_{1,2} \psi_{1,2}$-continuity reduces to a lot of existing continuity notions for some particular choices of the operations involved. Of course, there are a lot of other possible cases that can still be considered. We have the following table. 
TABLE 2.1

\begin{tabular}{|c|c|c|c|c|}
\hline Operation & Operation & Operation & Operation & Continuity type \\
\hline$\varphi_{1}$ & $\varphi_{2}$ & $\psi_{1}$ & $\psi_{2}$ & $\varphi_{1,2} \psi_{1,2}$-continuous \\
\hline int & $I$ & int & $I$ & continuous \\
\hline int & $\mathrm{cl}$ & int & $\mathrm{cl}$ & $\theta$-continuous [6] \\
\hline int & int $\circ \mathrm{cl}$ & int & int $\circ \mathrm{cl}$ & $\delta$-continuous [16] \\
\hline int & $I$ & int & $\mathrm{cl}$ & weakly-continuous [9] \\
\hline int & $\mathrm{cl}$ & int & $I$ & strongly $\theta$-continuous [16] \\
\hline int & $I$ & int & int $\circ \mathrm{cl}$ & almost continuous [19] \\
\hline int & $\mathrm{cl}$ & int & int $\circ \mathrm{cl}$ & almost strongly continuous [18] \\
\hline int & int $\circ \mathrm{cl}$ & int & $I$ & super continuous [14] \\
\hline int & int $\circ \mathrm{cl}$ & int & $\mathrm{cl}$ & weakly $\theta$-continuous \\
\hline cloint & $I$ & int & $I$ & semi-continuous [10] \\
\hline cloint & $I$ & $\mathrm{cl} \circ$ int & $I$ & irresolute [4] \\
\hline cloint & $\mathrm{scl}$ & $\mathrm{cl} \circ$ int & $\mathrm{scl}$ & semi-irresolute \\
\hline cloint & $\mathrm{scl}$ & cloint & $I$ & strongly irresolute \\
\hline int $\circ \mathrm{cl} \circ$ int & $I$ & int & $I$ & $\alpha$-continuous [13] \\
\hline int $\circ \mathrm{cl}$ & $I$ & int & $I$ & pre-continuous [12] \\
\hline $\mathrm{cl} \circ$ int $\circ \mathrm{cl}$ & $I$ & int & $I$ & $\beta$-continuous [1] \\
\hline $\mathrm{cl} \circ$ int & $I$ & int & $\mathrm{cl}$ & weakly semi-continuous \\
\hline int $\circ \mathrm{cl}$ & $I$ & int & $I$ & weakly pre-continuous \\
\hline cloint & $\mathrm{scl}$ & int & $\mathrm{cl}$ & $\theta$-semi-continuous [3] \\
\hline $\mathrm{cl} \circ$ int & $I$ & int & int $\circ \mathrm{cl}$ & almost semi-continuous \\
\hline $\mathrm{cl} \circ$ int & $\mathrm{cl}$ & int & $I$ & semi-strongly $\theta$-continuous \\
\hline int $\circ \mathrm{cl} \circ$ int & $I$ & int & $\mathrm{cl}$ & weakly $\alpha$-continuous \\
\hline int & $I$ & int $\circ \mathrm{cl}$ & $\mathrm{cl}$ & $\theta$-strongly continuous \\
\hline cloint & $I$ & cloint & $\mathrm{scl}$ & quasi-irresolute \\
\hline int $\circ \mathrm{cl} \circ$ int & $I$ & int $\circ \mathrm{cl} \circ$ int & $I$ & $\alpha$-irresolute [11] \\
\hline cloint & $I$ & int & $\mathrm{scl}$ & semi-weakly continuous [17] \\
\hline
\end{tabular}

The following theorem characterizes $\varphi_{1,2} \psi_{1,2}$-continuous functions in terms of the $\varphi_{1,2} \psi_{1,2}$-interior, $\varphi_{1,2} \psi_{1,2}$-closure, and $\varphi_{1,2} \psi_{1,2}$-frontier of sets.

TheOrem 2.2. Let $(X, \tau),(Y, \vartheta)$ be topological spaces, and let $\varphi_{1}, \varphi_{2} \in O_{(X, \tau)}$, $\psi_{1}, \psi_{2} \in O_{(Y, 9)}, I \leq \psi_{2}$. Then the following statements are equivalent for a function $f:(X, \tau) \rightarrow(Y, \vartheta):$

(1) $f \varphi_{1,2} \psi_{1,2}$-continuous;

(2) $f^{-1}(V) \subseteq \varphi_{1,2}-\operatorname{int}\left(f^{-1}\left(V^{\psi_{2}}\right)\right)$ for each $V \in O_{\psi_{1}}(Y)$;

(3) for each $x \in X$ and $V \in N_{\psi_{1}}(Y, f(x))$ there exists $U \in N_{\varphi_{1}}(X, x)$ such that $U^{\varphi_{2}} \subseteq f^{-1}\left(V^{\psi_{2}}\right)$;

(4) $f^{-1}\left(V^{\psi_{2}}\right) \in N_{\varphi_{1,2}}(X, x)$ for each $x \in X$ and $V \in N_{\psi_{1}}(Y, f(x))$;

(5) $f\left(\varphi_{1,2}-\mathrm{cl} A\right) \subseteq \psi_{1,2}-\mathrm{cl}(f(A))$ for every $A \subseteq X$;

(6) $\varphi_{1,2}-\mathrm{cl}\left(f^{-1}(B)\right) \subseteq f^{-1}\left(\psi_{1,2}-\mathrm{cl}(B)\right)$ for every $B \subseteq Y$;

(7) $f^{-1}\left(\psi_{1,2}-\operatorname{int}(B)\right) \subseteq \varphi_{1,2}-\operatorname{int}\left(f^{-1}(B)\right)$ for every $B \subseteq Y$; 
(8) $\varphi_{1,2}-\partial\left(f^{-1}(B)\right) \subseteq f^{-1}\left(\psi_{1,2}-\partial(B)\right)$ for every $B \subseteq Y$;

(9) $f\left(\varphi_{1,2}-\partial(A)\right) \subseteq \psi_{1,2}-\partial(f(A))$ for every $A \subseteq X$;

(10) $f\left(\varphi_{1,2}-\partial(A)\right) \subseteq \psi_{1,2}-\operatorname{cl}(f(A))$ for every $A \subseteq X$.

Proof. (1) $\Rightarrow(2)$. Let $V \in O_{\psi_{1}}(Y)$ and $x \in f^{-1}(V)$. Then $f(x) \in V$ and, by (1), there exists $U \in O_{\varphi_{1}}(X, x)$ such that $f\left(U^{\varphi_{2}}\right) \subseteq V^{\psi_{2}}$ and hence $U^{\varphi_{2}} \subseteq f^{-1}\left(V^{\psi_{2}}\right)$. Therefore, $x \in \varphi_{1,2}$-int $\left(f^{-1}\left(V^{\psi_{2}}\right)\right)$.

(2) $\Rightarrow(3)$. Let $x \in X$ and $V \in N_{\psi_{1}}(Y, f(x))$. Then, there exists $T \in O_{\psi_{1}}(Y, f(x))$ such that $T^{\psi_{2}} \subseteq V^{\psi_{2}}$. By (2), we have $f^{-1}(T) \subseteq \varphi_{1,2}-\operatorname{int}\left(f^{-1}\left(T^{\psi_{2}}\right)\right)$. Because $x \in f^{-1}(T)$, we have $x \in \varphi_{1,2}-\operatorname{int}\left(f^{-1}\left(T^{\psi_{2}}\right)\right)$. By definition of $\varphi_{1,2}$-interior, there exists $U \in O_{\varphi_{1}}(X, x)$ such that $U^{\varphi_{2}} \subseteq f^{-1}\left(T^{\psi_{2}}\right) \subseteq f^{-1}\left(V^{\psi_{2}}\right)$ and hence $U^{\varphi_{2}} \subseteq f^{-1}\left(V^{\psi_{2}}\right)$.

(3) $\Rightarrow(4)$. Let $x \in X$ and $V \in N_{\psi_{1}}(Y, f(x))$. By (3), there exists $U \in N_{\varphi_{1}}(X, x)$ such that $U^{\varphi_{2}} \subseteq f^{-1}\left(V^{\psi_{2}}\right)$. So, we have $f^{-1}\left(V^{\psi_{2}}\right) \in N_{\varphi_{1,2}}(X, x)$.

(4) $\Rightarrow(5)$. Suppose that $A \subseteq X$ and $f(x) \notin \psi_{1,2}-\operatorname{cl}(f(A))$. Then, there exists $V \in$ $N_{\psi_{1}}(Y, f(x))$ such that $f(A) \cap V^{\psi_{2}}=\varnothing$. So, we have $f^{-1}(f(A)) \cap f^{-1}\left(V^{\psi_{2}}\right)=\varnothing$ and hence $A \cap f^{-1}\left(V^{\psi_{2}}\right)=\varnothing$. Since, by (4), $f^{-1}\left(V^{\psi_{2}}\right) \in N_{\varphi_{1,2}}(X, x)$, then there exists $U \in O_{\varphi_{1}}(X, x)$ such that $U^{\varphi_{2}} \subseteq f^{-1}\left(V^{\psi_{2}}\right)$. So, we have $A \cap U^{\varphi_{2}}=\varnothing$ and hence $x \notin \varphi_{1,2}-\operatorname{cl}(A)$. Therefore $f(x) \notin f\left(\psi_{1,2}-\mathrm{cl}(A)\right)$.

(5) $\Rightarrow(6)$. Let $B \subseteq Y$. Because $f\left(f^{-1}(B)\right) \subseteq B$, we have $\psi_{1,2}-\operatorname{cl}\left(f\left(f^{-1}(B)\right)\right) \subseteq \psi_{1,2}-\operatorname{cl}(B)$. By (5), $f\left(\varphi_{1,2}-\operatorname{cl}\left(f^{-1}(B)\right)\right) \subseteq \psi_{1,2}-\operatorname{cl}\left(f\left(f^{-1}(B)\right)\right) \subseteq \psi_{1,2}-\operatorname{cl}(B)$. By applying $f^{-1}$ to all sides of inclusion, we get $\varphi_{1,2}-\operatorname{cl}\left(f^{-1}(B)\right) \subseteq f^{-1}\left(\psi_{1,2}-\operatorname{cl}(B)\right)$.

(6) $\Rightarrow(7)$. Let $B \subseteq Y$ and $x \in f^{-1}\left(\psi_{1,2}\right.$-int $\left.(B)\right)$. Then, $x \notin X \backslash f^{-1}\left(\psi_{1,2}-\operatorname{int}(B)\right)=$ $f^{-1}\left(Y \backslash \psi_{1,2}-\operatorname{int}(B)\right)=f^{-1}\left(\psi_{1,2}-\operatorname{cl}(Y \backslash B)\right)$, and hence $x \notin f^{-1}\left(\psi_{1,2}-\operatorname{cl}(Y \backslash B)\right)$. By (6), $\varphi_{1,2}-\operatorname{cl}\left(f^{-1}(Y \backslash B)\right) \subseteq f^{-1}\left(\psi_{1,2}-\operatorname{cl}(Y \backslash B)\right)$. Then $x \notin \varphi_{1,2}-\operatorname{cl}\left(f^{-1}(Y \backslash B)\right)=\varphi_{1,2}-\operatorname{cl}(X \backslash$ $\left.f^{-1}(B)\right)=X \backslash \varphi_{1,2}-\operatorname{int}\left(f^{-1}(B)\right)$, and hence $x \in \varphi_{1,2}-\operatorname{int}\left(f^{-1}(B)\right)$.

(7) $\Rightarrow(1)$. Let $x \in X$, and $V \in N_{\psi_{1}}(Y, f(x))$. Since $V \subseteq V^{\psi_{2}}$, we have $f(x) \in$ $\psi_{1,2}-\operatorname{int}\left(V^{\psi_{2}}\right)$, and hence $x \in f^{-1}\left(\psi_{1,2}-\operatorname{int}\left(V^{\psi_{2}}\right)\right)$. By (7), $x \in \varphi_{1,2}-\operatorname{int}\left(f^{-1}(B)\right)$. By the definition of $\varphi_{1,2}$-int, there exists $U \in O_{\varphi_{1}}(X, x)$ such that $U^{\varphi_{2}} \subseteq f^{-1}\left(V^{\psi_{2}}\right)$. So, we have $f\left(U^{\varphi_{2}}\right) \subseteq V^{\psi_{2}}$.

$(6) \Rightarrow(8)$. Let $B \subseteq Y$. We have

$$
\begin{aligned}
& \varphi_{1,2}-\partial\left(f^{-1}(B)\right)=\varphi_{1,2}-\mathrm{cl}\left(f^{-1}(B)\right) \cap \varphi_{1,2-\operatorname{cl}}\left(X \backslash f^{-1}(B)\right) \\
& =\varphi_{1,2}-\operatorname{cl}\left(f^{-1}(B)\right) \cap \varphi_{1,2}-\operatorname{cl}\left(f^{-1}(Y \backslash B)\right) \\
& \subseteq f^{-1}\left(\psi_{1,2^{-}}-\mathrm{cl}(B)\right) \cap f^{-1}\left(\psi_{1,2}-\mathrm{cl}(Y \backslash B)\right) \\
& =f^{-1}\left(\psi_{1,2}-\partial(B)\right) \text {. }
\end{aligned}
$$

(8) $\Rightarrow(9)$. Let $A \subseteq X$, then $A \subseteq f^{-1}(f(A))$, we get $\varphi_{1,2}-\partial(A) \subseteq \varphi_{1,2}-\partial\left(f^{-1}(f(A))\right) \subseteq$ $f^{-1}\left(\psi_{1,2}-\partial(f(A))\right)$, and hence $\varphi_{1,2^{-}} \partial(A) \subseteq f^{-1}\left(\psi_{1,2}-\partial(f(A))\right)$. This gives $f\left(\varphi_{1,2^{-}} \partial(A)\right)$ $\subseteq \psi_{1,2}-\partial(f(A))$.

(9) $\Rightarrow(5)$. Let $A \subseteq X$ and $y \in f\left(\varphi_{1,2}-\operatorname{cl}(A)\right)$. There exists $x \in \varphi_{1,2}-\operatorname{cl}(A)$ such that $f(x)=y$. Since $x \in \varphi_{1,2}-\operatorname{cl}(A)=\varphi_{1,2}-\partial(A) \cup \varphi_{1,2}-\operatorname{int}(A)$, then $x \in \varphi_{1,2}-\partial(A)$ or $x \in$ $\varphi_{1,2}$-int $(A)$. So, we have $y=f(x) \in f\left(\varphi_{1,2}-\partial(A)\right)$ or $y=f(x) \in f\left(\varphi_{1,2}\right.$-int $\left.(A)\right)$.

On the other hand, $f\left(\varphi_{1,2}-\operatorname{cl}(A)\right)=f\left(\varphi_{1,2}-\partial(A) \cup \varphi_{1,2}-\operatorname{int}(A)\right) \subseteq \psi_{1,2}-\partial(f(A)) \cup$ $f(A) \subseteq \psi_{1,2}-\operatorname{cl}(f(A)) \cup \psi_{1,2}-\operatorname{cl}(f(A))=\psi_{1,2}-\operatorname{cl}(f(A))$. Therefore, $f\left(\varphi_{1,2}-\operatorname{cl}(A)\right) \subseteq$ $\psi_{1,2}-\operatorname{cl}(f(A))$. 
(5) $\Rightarrow(10)$. Let $A \subseteq X$. By $\varphi_{1,2}-\partial(A) \subseteq \varphi_{1,2}-\operatorname{cl}(A)$, we have $f\left(\varphi_{1,2}-\partial(A)\right) \subseteq f\left(\varphi_{1,2^{-}}-\mathrm{cl}(A)\right)$ $\subseteq \psi_{1,2}-\mathrm{cl}(f(A))$, and hence $f\left(\varphi_{1,2}-\partial(A)\right) \subseteq \psi_{1,2}-\operatorname{cl}(f(A))$.

$(10) \Rightarrow(5)$. Let $A \subseteq X$. By $\varphi_{1,2^{-}} \operatorname{cl}(A)=\varphi_{1,2}-\partial(A) \cup \varphi_{1,2}$-int $(A)$, we get $f\left(\varphi_{1,2}-\operatorname{cl}(A)\right)$ $=f\left(\varphi_{1,2}-\partial(A) \cup \varphi_{1,2}-\operatorname{int}(A)\right)=f\left(\varphi_{1,2}-\partial(A)\right) \cup f\left(\varphi_{1,2}-\operatorname{int}(A)\right) \subseteq \psi_{1,2^{-}}-\operatorname{cl}(f(A)) \cup f\left(\varphi_{1,2^{-}}\right.$ $\operatorname{int}(A))=\psi_{1,2}-\operatorname{cl}(f(A))$.

Therefore, $f\left(\varphi_{1,2}-\operatorname{cl}(A)\right) \subseteq \psi_{1,2}-\operatorname{cl}(f(A))$.

COROLlary 2.3. If $f:(X, \tau) \rightarrow(Y, \vartheta)$ is $\varphi_{1,2} \psi_{1,2}$-continuous, then $f:\left(X, O_{\varphi_{1,2}}(X)\right)$ $\rightarrow\left(Y, O_{\psi_{1,2}}(Y)\right)$ is continuous.

Proof. Let $x \in X$ and $V \in O_{\psi_{1,2}}(Y, f(x))$. Then $x \in f^{-1}(V)$, and there exists a $W \in O_{\psi_{1}}(Y, f(x))$ such that $W^{\psi_{2}} \subseteq V$. Since $f$ is $\varphi_{1,2} \psi_{1,2}$-continuous at $x$, for $W \in$ $O_{\psi_{1}}(Y, f(x))$ there exists a $U \in O_{\varphi_{1}}(X, x)$ such that $U^{\varphi_{2}} \subseteq f^{-1}\left(W^{\psi_{2}}\right) \subseteq f^{-1}(V)$. If $x \in \varphi_{1,2}$-int $\left(f^{-1}(V)\right)$, then $f^{-1}(V) \in O_{\varphi_{1,2}}(X, x)$.

The reverse implication of the corollary is not true.

EXAMPLE 2.4. Let $(X, \tau),(Y, \vartheta)$ be topological spaces defined by $X=\{a, b, c\}$, $\tau=\{\phi, X,\{a\},\{c\},\{a, c\}\}, Y=\{a, b, c\}, \vartheta=\{\phi, X,\{b\}\}$, and $\varphi_{1}, \varphi_{2} \in O_{(X, \tau)}$ defined by $\varphi_{1}=\tau$-int, and for any $A \in P(X)$, we have

$$
A^{\varphi_{2}}= \begin{cases}\{a, b\}, & A=\{a\} \\ A, & \text { otherwise }\end{cases}
$$

$\psi_{1,} \psi_{2} \in O_{(Y, 9)}$ defined by $\psi_{1}=\vartheta$-int, and for any $B \in P(Y)$, we have

$$
B^{\psi_{2}}= \begin{cases}\{b, c\}, & B=\{b\} \\ B, & \text { otherwise. }\end{cases}
$$

Then $O_{\varphi_{1,2}}(X)=\{\phi, X,\{c\}\}$ and $O_{\psi_{1,2}}(Y)=\{\phi, Y\}$. Let $I$ be identity function. Because $I^{-1}\left(\{b\} \psi_{2}\right)=\{b, c\} \notin N_{\varphi_{1,2}}(X, b), I$ is not $\varphi_{1,2} \psi_{1,2}$-continuous but $I:\left(X, O_{\varphi_{1,2}}(X)\right)$ $\rightarrow\left(Y, O_{\psi_{1,2}}(Y)\right)$ is continuous.

To give a sufficient condition that the converse implication of Corollary 2.3 is true, we need to give the definition of $\varphi_{1,2}-T_{2}$ space.

DefinITION 2.5. Let $(X, \tau)$ be a topological space and $\varphi_{1,} \varphi_{2} \in O_{(X, \tau)}$, then $(X, \tau)$ is

(i) $\varphi_{1,2}-T_{1}$ space if and only if $\{x\}$ is $\varphi_{1,2}$-closed for each $x \in X$,

(ii) $\varphi_{1,2}-T_{2}$ space if and only if any distinct points $x, y$ of $X$ have $\varphi_{1}$-neighborhoods $U \in N_{\varphi_{1}}(X, x), V \in N_{\varphi_{1}}(X, y)$, and $U^{\varphi_{2}} \cap V^{\varphi_{2}}=\varnothing$ (see [7]).

THEOREM 2.6. Let $(X, \tau),(Y, \vartheta)$ be topological spaces, $(Y, \vartheta)$ is $\varphi_{1,2}-T_{2}$ space, and $I<\psi_{2}$. Then $f:(X, \tau) \rightarrow(Y, \vartheta)$ is $\varphi_{1,2} \psi_{1,2}$-continuous if and only if $f:\left(X, O_{\varphi_{1,2}}(X)\right) \rightarrow$ $\left(Y, O_{\psi_{1,2}}(Y)\right)$ is continuous.

Proof. $(\Rightarrow)$. Proved.

$(\Leftarrow)$. Let $f:\left(X, O_{\varphi_{1,2}}(X)\right) \rightarrow\left(Y, O_{\psi_{1,2}}(Y)\right)$ be continuous, $x \in X$, and $V \in N_{\varphi_{1}}(Y$, $f(x))$. Since $(Y, \vartheta)$ is $\varphi_{1,2}-T_{2}$ space, we have $V \in O_{\varphi_{1,2}}(Y, f(x))$. By hypothesis, $f^{-1}(V)$ $\in O_{\varphi_{1,2}}(X)$. Since $x \in f^{-1}(V)$, there exists $U \in N_{\varphi_{1}}(X, x)$ such that $U^{\varphi_{2}} \subseteq f^{-1}(V)$ 
and hence $U^{\varphi_{2}} \subseteq f^{-1}(V) \subseteq f^{-1}\left(V^{\psi_{2}}\right)$, and we have $f\left(U^{\varphi_{2}}\right) \subseteq V^{\psi_{2}}$. So $f$ is $\varphi_{1,2} \psi_{1,2^{-}}$ continuous.

TheOrem 2.7. Let $(X, \tau),(Y, 9)$ be topological spaces, $I=\psi_{2}$. Then the following statements are equivalent:

(1) $f:(X, \tau) \rightarrow(Y, \vartheta)$ is $\varphi_{1,2} \psi_{1,2}$-continuous;

(2) $f^{-1}(V) \in O_{\varphi_{1,2}}(X)$ for every $V \in O_{\psi_{1}}(Y)$;

(3) $f^{-1}(K) \in C_{\varphi_{1,2}}(X)$ for every $K \in C_{\psi_{1}}(Y)$.

Proof. (1) $\Rightarrow(2)$. Let $f$ be $\varphi_{1,2} \psi_{1,2}$-continuous, $V \in O_{\varphi_{1}}(Y)$, and $x \in f^{-1}(V)$. Since $f(x) \in V$ and $f$ is $\varphi_{1,2} \psi_{1,2}$-continuous, there exists $U \in N_{\varphi_{1}}(X, x)$ such that $U^{\varphi_{2}} \in$ $f^{-1}\left(V^{\psi_{2}}\right)=f^{-1}(V)$. So, we have $x \in \varphi_{1,2}-\operatorname{int}\left(f^{-1}(V)\right)$. Therefore, $f^{-1}(V) \subseteq \varphi_{1,2^{-}}$ $\operatorname{int}\left(f^{-1}(V)\right)$, and hence $f^{-1}(V) \in O_{\varphi_{1,2}}(X)$.

(2) $\Rightarrow(1)$. Let $x \in X$ and $V \in O_{\varphi_{1}}(Y, f(x))$. By (2), $f^{-1}(V) \in O_{\varphi_{1,2}}(X, x)$, and there exists $U \in N_{\varphi_{1}}(X, x)$ such that $U^{\varphi_{2}} \subseteq f^{-1}(V)=f^{-1}\left(V^{\psi_{2}}\right)$.

(2) $\Rightarrow(3)$. Let $K \in C_{\psi_{1}}(Y)$, then $Y \backslash K \in O_{\varphi_{1}}(Y)$. By hypothesis, we have $f^{-1}(Y \backslash K)=$ $X \backslash f^{-1}(K) \in O_{\varphi_{1,2}}(X)$. This implies $f^{-1}(K) \in C_{\varphi_{1,2}}(X)$.

$(3) \Rightarrow(2)$. It can be easily seen.

DEFINITION 2.8. Let $(X, \tau)$ be a topological space. An operation $\varphi \in O_{(X, \tau)}$ is said to be regular with respect to the family $\Omega \subseteq P(X)$, if for each $x \in X$ and $U, V \in \Omega$ containing $x$ there exists $W \in \Omega$ containing $x$ such that $W^{\varphi} \subseteq U^{\varphi} \cap V^{\varphi}$ (see [7]).

An operator $\varphi_{2} \in O_{(X, \tau)}$ is said to be $\varphi_{1,2}$-regular if and only if $\Omega=O \varphi_{1}(X)$, where $\varphi_{1} \in O_{(X, \tau)}$.

THEOREM 2.9. Let $(X, \tau),(Y, \vartheta)$ be topological spaces. Let $\varphi_{1}, \varphi_{2} \in O_{(X, \tau)}$ and $\varphi_{2} \in O_{(X, \tau)}$ be a $\varphi_{1,2}$-regular operator. Then for any function $f:(X, \tau) \rightarrow(Y, \vartheta)$, $f$ is $\varphi_{1,2} \psi_{1,2}$-continuous if and only if for each $x \in X, W \in N_{\varphi_{1}}(X, x)$, and $V \in$ $N_{\psi_{1}}(Y, f(x))$, there exists $U \in N_{\varphi_{1}}(X, x)$ such that $U^{\varphi_{2}} \subseteq W^{\varphi_{2}} \cap f^{-1}\left(V^{\psi_{2}}\right)$.

Proof. $(\Leftrightarrow)$. Let $x \in f^{-1}\left(\psi_{1,2}-\operatorname{int}(B)\right)$, for any $B \subseteq Y$. Then we have $f(x) \in \psi_{1,2}-\operatorname{int}(B)$. By definition of $\psi_{1,2}$-interior, there exists $G \in O_{\psi_{1}}(Y, f(x))$ such that $f(x) \in G^{\psi_{2}} \subseteq B$ and hence $x \in f^{-1}\left(G^{\psi_{2}}\right)$. So, we have $x \in W^{\varphi_{2}} \cap f^{-1}\left(V^{\psi_{2}}\right)$, for all $W \in N_{\varphi_{1}}(X, x)$. By hypothesis, there exists $U \in N_{\varphi_{1}}(X, x)$ such that $x \in U^{\varphi_{2}} \subseteq W^{\varphi_{2}} \cap f^{-1}\left(V^{\psi_{2}}\right) \subseteq$ $f^{-1}\left(V^{\psi_{2}}\right) \subseteq f^{-1}(B)$, and hence $x \in \varphi_{1,2}-\operatorname{int}\left(f^{-1}(B)\right)$. Therefore, $f^{-1}\left(\psi_{1,2}-\operatorname{int}(B)\right) \subseteq$ $\varphi_{1,2}-\operatorname{int}\left(f^{-1}(B)\right)$.

$(\Rightarrow)$. Let $V \in N_{\psi_{1}}(Y, f(x))$. There exists $T \in O_{\psi_{1}}(Y, f(x))$ such that $f(x) \in T^{\psi_{2}} \subseteq$ $V \subseteq V^{\psi_{2}}$ and hence $f(x) \in \psi_{1,2}-\operatorname{int}\left(V^{\psi_{2}}\right)$. Therefore, $x \in f^{-1}\left(\psi_{1,2}-\operatorname{int}\left(V^{\psi_{2}}\right)\right)$. By $\varphi_{1,2} \psi_{1,2}$-continuity of $f, x \in \varphi_{1,2}$-int $\left(f^{-1}\left(V^{\psi_{2}}\right)\right)$. By definition of $\varphi_{1,2}$-interior, there exists $U \in O_{\varphi_{1}}(X, x)$ such that $x \in U^{\varphi_{2}} \subseteq f^{-1}\left(V^{\psi_{2}}\right)$. Since $\varphi_{2}$ is $\varphi_{1,2}$-regular operator, there exists $U^{*} \in O_{\varphi_{1}}(X, x)$ such that $x \in U^{* \varphi_{2}} \subseteq U^{\varphi_{2}} \cap f^{-1}\left(V^{\psi_{2}}\right) \subseteq W^{\varphi_{2}} \cap f^{-1}\left(V^{\psi_{2}}\right)$.

The following theorem characterizes the set of $\varphi_{1,2} \psi_{1,2}$-noncontinuous points denoted by $\varphi_{1,2} \psi_{1,2}$ - NC $(f)$ of any function $f$.

THEOREM 2.10. Let $(X, \tau),(Y, \vartheta)$ be topological spaces, and let $f:(X, \tau) \rightarrow(Y, \vartheta)$ be any function, $\varphi_{1}, \varphi_{2} \in O_{(X, \tau)}, \psi_{1}, \psi_{2} \in O_{(Y, 9)}$, and $I=\psi_{2}$. Then $\varphi_{1,2} \psi_{1,2}-\mathrm{NC}(f)=$ $\cup\left\{f^{-1}(V) \cap \varphi_{1,2}-\partial\left(f^{-1}(V)\right) \mid V \in O_{\psi_{1}}(Y)\right\}$. 
Proof. Let $x \in \varphi_{1,2} \psi_{1,2}$ - NC $(f)$. Then $f$ is not continuous at $x$. So there exits $W \in O_{\psi_{1}}(Y, f(x))$ such that $f\left(U^{\varphi_{2}}\right) \nsubseteq W^{\varphi_{2}}$ for each $U \in O_{\varphi_{1}}(X, x)$. Hence for each $U \in O_{\varphi_{1}}(X, x)$, we have $\varnothing \neq f\left(U^{\varphi_{2}}\right) \cap(Y-W)=f\left(U^{\varphi_{2}} \cap f^{-1}(Y-W)\right)=f\left(U^{\varphi_{2}} \cap(X-\right.$ $\left.f^{-1}(W)\right)$ ). Therefore, we get $U^{\varphi_{2}} \cap\left(X-f^{-1}(W)\right) \neq \varnothing$ and hence $x \in \varphi_{1,2}-\operatorname{cl}\left(X \backslash f^{-1}(W)\right)$. On the other hand, $x \in \varphi_{1,2}-\operatorname{cl}\left(X \backslash f^{-1}(W)\right) \cap f^{-1}(W)=f^{-1}(W) \cap \varphi_{1,2}-\partial\left(f^{-1}(W)\right) \subseteq$ $\cup\left\{f^{-1}(V) \cap \varphi_{1,2}-\partial\left(f^{-1}(V)\right) \mid V \in O_{\psi_{1}}(Y)\right\}$. Finally, we get $x \in \cup\left\{f^{-1}(V) \cap \varphi_{1,2^{-}}\right.$ $\left.\partial\left(f^{-1}(V)\right) \mid V \in O_{\psi_{1}}(Y)\right\}$. This shows that $\varphi_{1,2} \psi_{1,2}-\mathrm{NC}(f) \subseteq \cup\left\{f^{-1}(V) \cap \varphi_{1,2^{-}}\right.$ $\left.\partial\left(f^{-1}(V)\right) \mid V \in O_{\psi_{1}}(Y)\right\}$.

To show the reverse inclusion, let $x \in \cup\left\{f^{-1}(V) \cap \varphi_{1,2}-\partial\left(f^{-1}(V)\right) \mid V \in O_{\psi_{1}}(Y)\right\}$. There exists $V \in O_{\psi_{1}}(Y)$ such that $x \in f^{-1}(V) \cap \varphi_{1,2}-\partial\left(f^{-1}(V)\right)=f^{-1}(V) \backslash\left(\varphi_{1,2^{-}}\right.$ $\left.\operatorname{int}\left(f^{-1}(V)\right)\right)$, and hence $x \notin \varphi_{1,2}-\operatorname{int}\left(f^{-1}(V)\right)$. Suppose $f$ is $\varphi_{1,2} \psi_{1,2}$-continuous at $x$. Since $x \in f^{-1}(V)$ and $V \in O_{\psi_{1}}(Y)$, there exists $U \in O_{\varphi_{1}}(X, x)$ such that $U^{\varphi_{2}} \subseteq$ $f^{-1}\left(V^{\psi_{2}}\right)=f^{-1}(V)$, and hence $x \in \varphi_{1,2}-\operatorname{int}\left(f^{-1}(V)\right)$. This is a contradiction.

THEOREM 2.11. Let $(X, \tau),(Y, \vartheta)$ be topological spaces and let $\varphi_{1}, \varphi_{2}, \varphi_{3}, \varphi_{4} \in$ $O_{(X, \tau)}, \psi_{1}, \psi_{2}, \psi_{3}, \psi_{4} \in O_{(Y, 9)}$ be operators with the property $\varphi_{3} \geq \varphi_{1}, \varphi_{2} \geq \varphi_{4}, \psi_{1} \geq$ $\psi_{3}$, and $\psi_{4} \geq \psi_{2}$. Then for any function $f:(X, \tau) \rightarrow(Y, \vartheta), f$ is $\varphi_{1,2} \psi_{1,2}$-continuous if and only if $f$ is $\varphi_{3,4} \psi_{3,4}$-continuous.

Proof. Let $x \in X$ and $V \in O_{\psi_{3}}(Y, f(x))$. Since $V \subseteq V^{\psi_{3}} \subseteq V^{\psi_{1}}$ and hence $V \subseteq V^{\psi_{1}}$, we have $V \in O_{\psi_{1}}(Y, f(x))$, by $\varphi_{1,2} \psi_{1,2}$-continuity of $f$, there exists $U \in O_{\varphi_{1}}(X, x)$ such that $U^{\varphi_{2}} \subseteq f^{-1}\left(V^{\psi_{2}}\right) \subseteq f^{-1}\left(V^{\psi_{4}}\right)$. By $U^{\varphi_{4}} \subseteq U^{\varphi_{2}}$ and $V^{\psi_{2}} \subseteq V^{\psi_{4}}$, we have $U^{\varphi_{4}} \subseteq$ $U^{\varphi_{2}} \subseteq f^{-1}\left(V^{\psi_{2}}\right) \subseteq f^{-1}\left(V^{\psi_{4}}\right)$, and hence $U^{\varphi_{4}} \subseteq f^{-1}\left(V^{\psi_{4}}\right)$. Therefore, $f$ is $\varphi_{3,4} \psi_{3,4^{-}}$ continuous.

THEOREM 2.12. Let $(X, \tau)$ be a topological space, and let $\varphi_{1}, \varphi_{2}, \varphi_{3}, \varphi_{4} \in O_{(X, \tau)}$ be operators with the property $\varphi_{1} \geq \varphi_{3}, \varphi_{2} \geq \varphi_{4}$. Then the identity function $I:(X, \tau) \rightarrow$ $(Y, \vartheta)$ is $\varphi_{1,2}, \varphi_{3,4}$-continuous.

Proof. Let $x \in X$ and $V \in O_{\varphi_{3}}(Y, I(x))$. Since $V^{\varphi_{3}} \subseteq V^{\varphi_{1}}$, then we have $V \in$ $O_{\varphi_{1}}(Y, I(x))$. So we get $I\left(V^{\psi_{2}}\right)=V^{\psi_{2}} \subseteq V^{\psi_{4}}$ and hence $I\left(V^{\psi_{2}}\right) \subseteq V^{\psi_{4}}$. Therefore, $I$ is $\varphi_{1,2}, \varphi_{3,4}$-continuous.

The last two theorems give the following diagrams:

$$
\delta \text {-continuity } \Longrightarrow \theta \text {-continuity, }
$$

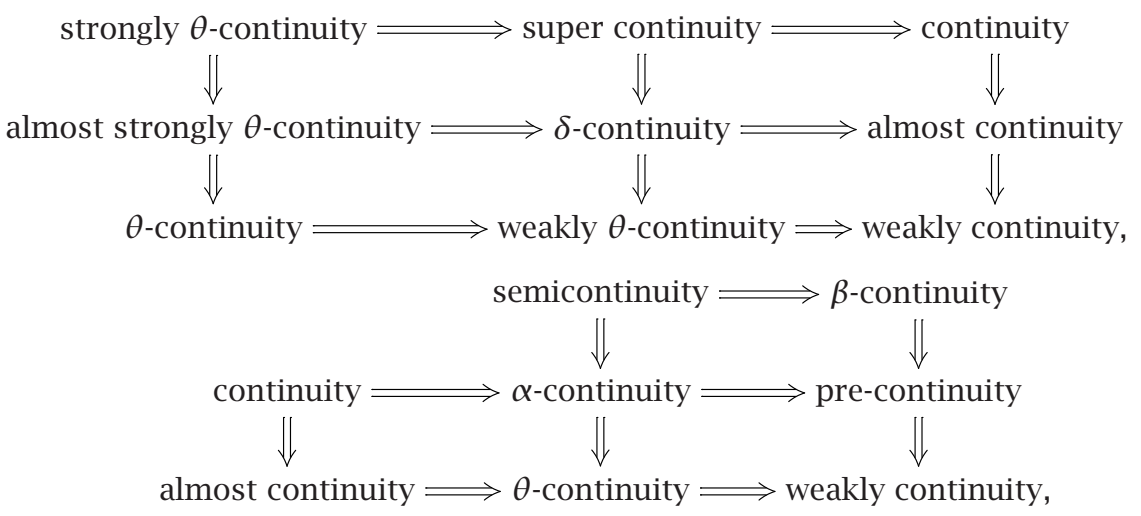



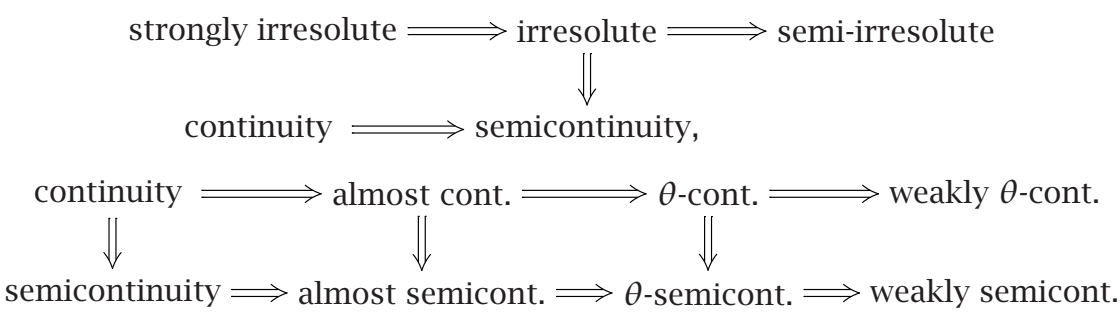

\section{Some properties of $\varphi_{1,2} \psi_{1,2}$-continuous functions}

THEOREM 3.1. Let $f:(X, \tau) \rightarrow(Y, 9)$ be a one-to-one and $\varphi_{1,2} \psi_{1,2}$-continuous function and let $(Y, 9)$ be a $\psi_{1,2}-T_{2}\left(\psi_{1,2}-T_{1}\right)$ topological space. Then $(X, \tau)$ is a $\varphi_{1,2}-T_{2}\left(\varphi_{1,2}-T_{1}\right)$ topological space.

Proof. We prove $(X, \tau)$ is a $\varphi_{1,2}-T_{2}$. The other case can be proved similarly. Let $x, y \in X$ and $x \neq y$. Since $f$ is a one-to-one function, we have $f(x) \neq f(y)$. Since $(Y, \vartheta)$ is a $\varphi_{1,2}-T_{2}$, then there exist $V_{1} \in N_{\psi_{1}}(Y, f(x)), V_{2} \in N_{\psi_{1}}(Y, f(y))$, and $V_{1}^{\psi_{2}} \cap V_{2}^{\psi_{2}}=$ $\varnothing$. Since $f$ is $\varphi_{1,2} \psi_{1,2}$-continuous, then there exist $U_{1} \in N_{\varphi_{1}}(X, x), U_{2} \in N_{\varphi_{1}}(X, y)$ such that $f\left(U_{1}^{\varphi_{2}}\right) \subseteq V_{1}^{\psi_{2}}$ and $f\left(U_{2}^{\varphi_{2}}\right) \subseteq V_{2}^{\psi_{2}}$ and $f\left(U_{1}^{\varphi_{2}}\right) \cap f\left(U_{2}^{\varphi_{2}}\right)=\varnothing$. Hence we have $U_{1}^{\varphi_{2}} \cap U_{2}^{\varphi_{2}}=\varnothing$. Therefore $(X, \tau)$ is a $\varphi_{1,2}-T_{2}$.

THEOREM 3.2. The topological space $(X, \tau)$ is a $\varphi_{1,2}-T_{i}(i=1,2)$ if and only if for any $x, y \in X$ and $x \neq y$ there exists $a(Y, \vartheta)$ topological space with $\varphi_{1,2}-T_{2}$, a function $f$ from $(X, \tau)$ to $(Y, \vartheta)$ such that $\varphi_{1,2} \psi_{1,2}$-continuous, and $f(x) \neq f(y)$.

Proof. We prove the theorem for $i=2$. The other case can be proved similarly.

$(\Rightarrow)$. Suppose $(X, \tau)$ is a $\varphi_{1,2}-T_{2}$, it is easy to check that the identity function defined from $(X, \tau)$ to $(X, \tau)$ is the desired function $f$.

$(\Leftarrow)$. Let $x, y \in X$ and $x \neq y$. By hypothesis, there exists a $(Y, \vartheta)$ topological space with $\varphi_{1,2}-T_{2}$, an $f$ function from $(X, \tau)$ to $(Y, \vartheta)$ such that $\varphi_{1,2} \psi_{1,2}$-continuous and $f(x) \neq f(y)$. Since $(Y, 9)$ is $\varphi_{1,2}-T_{2}$ and $f(x) \neq f(y)$, then there exist $V_{1} \in N_{\psi_{1}}(Y, f(x))$, $V_{2} \in N_{\psi_{1}}(Y, f(y))$, and $V_{1}^{\psi_{2}} \cap V_{2}^{\psi_{2}}=\varnothing$. Since $f$ is $\varphi_{1,2} \psi_{1,2}$-continuous, then there exist $U_{1} \in N_{\varphi_{1}}(X, x), U_{2} \in N_{\varphi_{1}}(X, y)$ such that $f\left(U_{1}^{\varphi_{2}}\right) \subseteq V_{1}^{\psi_{2}}$ and $f\left(U_{2}^{\varphi_{2}}\right) \subseteq V_{2}^{\psi_{2}}$ and $f\left(U_{1}^{\varphi_{2}}\right) \cap f\left(U_{2}^{\varphi_{2}}\right)=\varnothing$. Hence we have $U_{1}^{\varphi_{2}} \cap U_{2}^{\varphi_{2}}=\varnothing$. Therefore $(X, \tau)$ is a $\varphi_{1,2}-T_{2}$.

Definition 3.3. Let $(X, \tau)$ be a topological space and $\varphi_{1}, \varphi_{2} \in O_{(X, \tau)}$.

(i) The family $\Omega \subseteq O_{\varphi_{1}}(X)$ is called a $\varphi_{1,2}$-open cover of a set $K$ if and only if $K \subseteq \cup\left\{U^{\varphi_{2}} \mid U \in \Omega\right\}$.

(ii) Let the family $\Omega \subseteq O_{\varphi_{1}}(X)$ be a $\varphi_{1,2}$-open cover of a set $K$. The subfamily $\Omega^{*}$ of $\Omega$ is called a $\varphi_{1,2}$-open subcover of the set $K$ if and only if $K \subseteq \cup\left\{U^{\varphi_{2}} \mid U \in \Omega^{*}\right\}$ (see [7]).

(iii) Any subset $K$ of $X$ is called $\varphi_{1,2}$-compact if and only if each $\varphi_{1,2}$-open cover of $K$ has a finite $\varphi_{1,2}$-open subcover of $K$.

(iv) The set $X$ is called $\varphi_{1,2}$-compact if and only if it is $\varphi_{1,2}$-compact (see [7]).

THEOREM 3.4. Let $f$ be a $\varphi_{1,2} \psi_{1,2}$-continuous function defined from $(X, \tau)$ to $(Y, 9)$ and $A \subseteq X$ be $\varphi_{1,2}$-compact. Then $f(A)$ is $\psi_{1,2}$-compact. 
PRoof. Let the family $\Omega_{f(A)}=\left\{V \in O_{\psi_{1}}(Y) \mid i \in \Delta\right\}$ be a $\psi_{1,2}$-open cover of $f(A)$ and $y \in f(A)$. There exist $x \in A$ and $i_{y} \in \Delta$ such that $y=f(x)$ and $y \in V_{i_{y}}$. Since $f$ is $\varphi_{1,2} \psi_{1,2}$-continuous, there exists $U_{i_{y}} \in N_{\varphi_{1}}(X, x)$ such that $f\left(U_{i_{y}}^{\varphi_{2}}\right) \subseteq V_{i_{y}}^{\psi_{2}}$ and hence $\Omega_{A}=\left\{U_{i_{y}} \mid y \in f(A), i \in \Delta\right\}$ is a $\varphi_{1}$-open cover of $A$. From $\varphi_{1,2}$-compactness of $A$, there exists a finite subfamily $\Delta_{\circ}$ of $\Delta$ such that $A \subseteq \bigcup_{i \in \Delta_{\circ}} U_{i_{y}}$. So the family $\Omega_{f(A)}^{*}=$ $\left\{V_{i_{y}} \mid i \in \Delta_{0}\right\} \subseteq \Omega_{f(A)}$ is a $\psi_{1,2}$-open cover of $f(A)$. Then $f(A)$ is $\psi_{1,2}$-compact.

THEOREM 3.5. Let $f:(X, \tau) \rightarrow(Y, \vartheta)$ be a $\varphi_{1,2} \psi_{1,2}$-continuous function and $(X, \tau)$ be a $\varphi_{1,2}$-compact space. Then $(Y, \vartheta)$ is $\psi_{1,2}$-compact.

Proof. The proof is immediate from Theorem 3.4.

DeFinITION 3.6 [7]. Let $(X, \tau)$ be a topological space and $\varphi_{1}, \varphi_{2} \in O_{(X, \tau)}$. A subset $D$ of $X$ is called $\varphi_{1,2}$-dense in $X$ if and only if $\varphi_{1,2}-\operatorname{cl}(D)=X$.

THEOREM 3.7. Let $f, g:(X, \tau) \rightarrow(Y, \vartheta)$ be $\varphi_{1,2} \psi_{1,2}$-continuous functions and $\varphi_{2}$ regular with respect to $O_{\varphi_{1}}(X)$ and let $(Y, \vartheta)$ be $\psi_{1,2}-T_{2}$ space. If $D \subseteq X$ is a closed $\psi_{1,2}$-dense set and $f(x)=g(x)$ for each $x \in D$. Then $f=g$.

Proof. Suppose $x_{0} \in X$ and $x_{0} \notin D$. Then $f\left(x_{0}\right) \neq g\left(x_{0}\right)$. Since $(Y, \vartheta)$ is $\psi_{1,2}-T_{2}$, then there exist $V_{1} \in N_{\psi_{1}}\left(Y, f\left(x_{0}\right)\right), V_{2} \in N_{\psi_{1}}\left(Y, g\left(x_{0}\right)\right)$, and $V_{1}^{\psi_{2}} \cap V_{2}^{\psi_{2}}=\varnothing$. Since $f, g$ are $\varphi_{1,2} \psi_{1,2}$-continuous at $x_{0}$, then there exist $U_{1} \in N_{\varphi_{1}}\left(X, x_{0}\right), U_{2} \in N_{\varphi_{1}}\left(X, x_{0}\right)$ such that $f\left(U_{1}^{\varphi_{2}}\right) \subseteq V_{1}^{\psi_{2}}$ and $g\left(U_{2}^{\varphi_{2}}\right) \subseteq V_{2}^{\psi_{2}}$. By regularity of $\varphi_{2}$, there exists $U_{0} \in$ $N_{\varphi_{1}}\left(X, x_{0}\right)$ such that $U_{0}^{\varphi_{2}} \subseteq U_{1}^{\varphi_{2}} \cap U_{2}^{\varphi_{2}}$. So, by $V_{1}^{\psi_{2}} \cap V_{2}^{\psi_{2}}=\varnothing$, we have $U_{0}^{\varphi_{2}} \cap D=\varnothing$ and hence $x \notin \varphi_{1,2}-\operatorname{cl}(D)$. This contradicts the $\varphi_{1,2}$-closedness of $D$ and $D=\varphi_{1,2}-\operatorname{cl}(D)=$ $X$. So, we have $D=X$.

THEOREM 3.8. Let $\left(X_{i}, T_{i}\right),\left(Y_{i}, \vartheta_{i}\right)(i=1,2)$ be topological spaces, and $F=f_{1} \times f_{2}$ : $\left(X_{1} \times X_{2}, \tau_{1} \times \tau_{2}\right) \rightarrow\left(Y_{1} \times Y_{2}, \vartheta_{1} \times \vartheta_{2}\right), \varphi_{11}, \varphi_{12} \in O_{\left(X_{1}, \tau_{1}\right)}, \varphi_{21}, \varphi_{22} \in O_{\left(X_{2}, \tau_{2}\right)}$, and let $\psi_{11}, \psi_{12} \in O_{\left(Y_{1}, \vartheta_{1}\right)}, \psi_{21}, \psi_{22} \in O_{\left(Y_{2}, \vartheta_{2}\right)}$ be operators.

If $f_{1}:\left(X_{1}, \tau_{1}\right) \rightarrow\left(Y_{1}, \vartheta_{1}\right)$ is $\varphi_{11,12} \psi_{11,12}$-continuous and $f_{2}:\left(X_{2}, \tau_{2}\right) \rightarrow\left(Y_{2}, \vartheta_{2}\right)$ is $\varphi_{21,22} \psi_{21,22}$-continuous, then $F$ is a $\varphi_{1,2} \psi_{1,2}$-continuous function, where $\varphi_{1}, \varphi_{2} \in$ $O_{\left(X_{1} \times X_{2}, \tau_{1} \times \tau_{2}\right)}, \psi_{1}, \psi_{2} \in O_{\left(Y_{1} \times Y_{2}, 9_{1} \times 9_{2}\right)}$, and $\psi_{2}$ is monotonous and compatible with $\psi_{12}$ and $\psi_{22}$.

Proof. Let $(x, y) \in X_{1} \times X_{2}$ and $V \in O_{\psi_{1}}\left(Y_{1} \times Y_{2},\left(f_{1}(x), f_{2}(y)\right)\right)$. Then there exist $V_{1} \in O_{\psi_{11}}\left(Y_{1}, f_{1}(x)\right)$ and $V_{2} \in O_{\psi_{21}}\left(Y_{2}, f_{2}(y)\right)$ such that $\left(f_{1}(x), f_{2}(y)\right) \in V_{1} \times V_{2}$. Since $f_{1}$ is $\varphi_{11,12} \psi_{11,12}$-continuous and $f_{2}$ is $\varphi_{21,22} \psi_{21,22}$-continuous, then there exist $U_{1} \in O_{\varphi_{11}}\left(X_{1}, x\right)$ and $U_{2} \in O_{\varphi_{21}}\left(X_{2}, y\right)$ such that $U_{1}^{\varphi_{12}} \subseteq f_{1}^{-1}\left(V_{1}^{\psi_{12}}\right)$ and $U_{2}^{\varphi_{21}} \subseteq$ $f_{2}^{-1}\left(V_{2}^{\psi_{21}}\right)$. Since $U_{1} \times U_{2} \in O_{\varphi_{1}}\left(X_{1} \times X_{2},(x, y)\right)$, we have $F\left(U_{1}^{\varphi_{12}} \times U_{2}^{\varphi_{21}}\right)=f_{1}\left(U_{1}^{\varphi_{12}}\right) \times$ $f_{2}\left(U_{2}^{\varphi_{21}}\right) \subseteq V_{1}^{\psi_{12}} \times V_{2}^{\psi_{21}} \subseteq V^{\psi_{2}}$. Therefore, $F$ is a $\varphi_{1,2} \psi_{1,2}$-continuous function.

TheOrem 3.9. Let $(X, \tau),(Y, \vartheta)$, and let $(Z, \varpi)$ be topological spaces and let $f_{1}$ : $(X, \tau) \rightarrow(Y, \vartheta), f_{2}:(X, \tau) \rightarrow(Z, \varpi)$ be any functions. Let $f:(X, \tau) \rightarrow(Y \times Z, \tau \times$ $\varpi)$ be a function defined by $f(x)=\left(f_{1}(x), f_{2}(x)\right)$ for each $x \in X$, and let $\varphi_{1}, \varphi_{2} \in$ $O_{(X, \tau)}, \psi_{1}, \psi_{2} \in O_{(Y, 9)}, \gamma_{1}, \gamma_{2} \in O_{(Z, \varpi)}$, and $\xi_{1}, \xi_{2} \in O_{(Y \times Z, \tau \times \varpi)}$ be operators. Then the following statements are true:

(i) if $A \subseteq X, B \subseteq Y, C \subseteq Z$, and $f(A) \subseteq B \times C$, then $f_{1}(A) \subseteq B$ and $f_{2}(A) \subseteq C$; 
(ii) if $T \in O_{\xi_{1}}(Y \times Z, f(x))$, then there exist $V \in O_{\psi_{1}}\left(Y, f_{1}(x)\right), W \in O_{\gamma_{1}}\left(Z, f_{2}(x)\right)$ such that $f(x) \in V \times W$ and $V^{\psi_{2}} \times W^{\gamma_{2}} \subseteq T^{\xi_{2}}$;

(iii) if $A_{1}, A_{2} \subseteq X, B \subseteq Y, C \subseteq Z$, and $f_{1}\left(A_{1}\right) \times f_{2}\left(A_{2}\right) \subseteq B \times C$, then $f\left(A_{1} \cap A_{2}\right) \subseteq$ $B \times C$.

THEOREM 3.10. Let $(X, \tau),(Y, \vartheta)$, and $(Z, \varpi)$ be topological spaces and $f_{1}:(X, \tau)$ $\rightarrow(Y, \vartheta)$ and $f_{2}:(X, \tau) \rightarrow(Z, \varpi)$ any functions. Let $f:(X, \tau) \rightarrow(Y \times Z, \tau \times \varpi)$ be a function defined by $f(x)=\left(f_{1}(x), f_{2}(x)\right)$ for each $x \in X$ and let $\varphi_{1}, \varphi_{2} \in O_{(X, \tau)}$, $\psi_{1}, \psi_{2} \in O_{(Y, 9)}, \gamma_{1}, \gamma_{2} \in O_{(Z, \varpi)}$, and $\xi_{1}, \xi_{2} \in O_{(Y \times Z, \tau \times \varpi)}$ be operators with the property $\xi_{2}$ compatible with $\psi_{2}$ and $\gamma_{2}$. Then $f$ is $\varphi_{1,2} \xi_{1,2}$-continuous if and only if $f_{1}$ is $\varphi_{1,2} \psi_{1,2}$ continuous and $f_{2}$ is $\varphi_{1,2} \gamma_{1,2}$-continuous.

Proof. $(\Rightarrow)$. Let $x \in X, V \in O_{\psi_{1}}\left(Y, f_{1}(x)\right)$, and $W \in O_{\gamma_{1}}\left(Z, f_{2}(x)\right)$. Then we have $V \times W \in O \xi_{1}(Y \times Z, f(x))$. By $\varphi_{1,2} \xi_{1,2}$-continuity of $f$, there exists $U \in O_{\varphi_{1}}\left(X_{1}, x\right)$ such that $f\left(U^{\varphi_{2}}\right) \subseteq(V \times W)^{\xi_{2}}=V^{\psi_{2}} \times W^{\gamma_{2}}$. By Theorem 3.9(i), we have $f_{1}\left(U^{\varphi_{2}}\right) \subseteq$ $V^{\psi_{2}}$ and $f_{2}\left(U^{\varphi_{2}}\right) \subseteq W^{\gamma_{2}}$, and hence $f_{1}$ is $\varphi_{1,2} \psi_{1,2}$-continuous and $f_{2}$ is $\varphi_{1,2} \gamma_{1,2^{-}}$ continuous.

$(\Leftarrow)$. Conversely, let $x \in X$ and $T \in O \xi_{1}(Y \times Z, f(x))$. By Theorem 3.9(ii), there exist $V \in O_{\psi_{1}}\left(Y, f_{1}(x)\right)$ and $W \in O_{\gamma_{1}}\left(Z, f_{2}(x)\right)$ such that $f(x) \in V \times W$ and $V^{\psi_{2}} \times W^{\gamma_{2}} \subseteq$ $T^{\xi_{2}}$. Since $f_{1}$ is $\varphi_{1,2} \psi_{1,2}$-continuous and $f_{2}$ is $\varphi_{1,2} \gamma_{1,2}$-continuous, then there exist $U_{1}, U_{2} \in O_{\varphi_{1}}\left(X_{1}, x\right)$ such that $f_{1}\left(U_{1}^{\varphi_{2}}\right) \subseteq V^{\psi_{2}}$ and $f_{2}\left(U_{2}^{\varphi_{2}}\right) \subseteq W^{\gamma_{2}}$, and hence $f_{1}\left(U_{1}^{\varphi_{2}}\right) \times$ $f_{2}\left(U_{2}^{\varphi_{2}}\right) \subseteq V^{\psi_{2}} \times W^{\gamma_{2}} \subseteq T^{\xi_{2}}$. By Theorem 3.9(iii), we have $f\left(\left(U_{1} \cap U_{2}\right)^{\varphi_{2}}\right) \subseteq T^{\xi_{2}}$. Thus $f$ is $\varphi_{1,2} \xi_{1,2}$-continuous.

THEOREM 3.11. Let $(X, \tau),(Y, \vartheta)$, and $(Z, \varpi)$ be topological spaces and $\varphi_{1}, \varphi_{2} \in$ $O_{(X, \tau)}, \psi_{1}, \psi_{2} \in O_{(Y, 9)}, \gamma_{1}, \gamma_{2} \in O_{(Z, \varpi)}$. If $f:(X, \tau) \rightarrow(Y, \vartheta)$ is $\varphi_{1,2} \psi_{1,2}$-continuous and $g:(Y, \vartheta) \rightarrow(Z, \varpi)$ is $\psi_{1,2} \gamma_{1,2}$-continuous function. Then $g \circ f:(X, \tau) \rightarrow(Z, \varpi)$ is $\varphi_{1,2} \gamma_{1,2}$-continuous.

Proof. Let $f:(X, \tau) \rightarrow(Y, \vartheta)$ be a $\varphi_{1,2} \psi_{1,2}$-continuous and $g:(Y, \vartheta) \rightarrow(Z, \varpi)$ a $\psi_{1,2} \gamma_{1,2}$-continuous function. Let any $x \in X$ and $W \in O_{\gamma_{1}}(Z,(g \circ f)(x))$. Since $g$ is $\psi_{1,2} \gamma_{1,2}$-continuous at $f(x)$, there is a $V \in O_{\psi_{1}}(Y, f(x))$ such that $g\left(V^{\psi_{2}}\right) \subseteq W^{\gamma_{2}}$. Since $f$ is $\varphi_{1,2} \psi_{1,2}$-continuous at $x$, there exists $U \in O_{\varphi_{1}}(X, x)$ such that $f\left(U^{\varphi_{2}}\right) \subseteq V^{\psi_{2}}$. Therefore, we obtain $(g \circ f)\left(U^{\varphi_{2}}\right)=g\left(f\left(U^{\varphi_{2}}\right)\right) \subseteq g\left(V^{\psi_{2}}\right) \subseteq W^{\gamma_{2}}$. This proves the $\varphi_{1,2} \gamma_{1,2}$-continuity of $g \circ f$ at $x$.

Definition 3.12 [7]. A topological space $(X, \tau)$ is called $\varphi_{1,2}$-hyperconnected if $X=U^{\varphi_{2}}$ for each $U \in O_{\varphi_{1}}(X)$.

THEOREM 3.13. If $f:(X, \tau) \rightarrow(Y, \vartheta)$ is a $\varphi_{1,2} \psi_{1,2}$-continuous surjection function and $(X, \tau)$ is $\varphi_{1,2}$-hyperconnected, then $(Y, \vartheta)$ is $\psi_{1,2}$-hyperconnected.

Proof. Let any $x \in X$ and $V \in O_{\psi_{1}}(Y, f(x))$. By $\varphi_{1,2} \psi_{1,2}$-continuity of $f$, there exists $U \in O_{\varphi_{1}}(X, x)$ such that $f\left(U^{\varphi_{2}}\right) \subseteq V^{\psi_{2}}$. Since $(X, \tau)$ is $\varphi_{1,2}$-hyperconnected, we have $Y=f(X)=f\left(U^{\varphi_{2}}\right) \subseteq V^{\psi_{2}}$ and hence $Y=V^{\psi_{2}}$.

\section{REFERENCES}

[1] M. E. Abd El-Monsef, S. N. El-Deeb, and R. A. Mahmoud, $\beta$-open sets and $\beta$-continuous mapping, Bull. Fac. Sci. Assiut Univ. A 12 (1983), no. 1, 77-90. 
[2] D. Andrejević, Semi-open sets, Mat. Vesnik 38 (1986), 24-32.

[3] S. P. Arya and M. P. Bhamini, Some weaker forms of semi-continuous functions, Ganita 33 (1982), no. 1-2, 124-134.

[4] S. G. Crossley and S. K. Hildebrand, Semi-topological properties, Fund. Math. 74 (1972), no. 3, 233-254.

[5] Á. Császár, Generalized open sets, Acta Math. Hungar. 75 (1997), no. 1-2, 65-87.

[6] S. Fomin, Extensions of topological spaces, Ann. of Math. (2) 44 (1943), 471-480.

[7] A. Kandil, E. E. Kerre, and A. A. Nouh, Operations and mappings on fuzzy topological spaces, Ann. Soc. Sci. Bruxelles Sér. I 105 (1991), no. 4, 167-188.

[8] J. K. Kohli, A framework including the theories of continuous functions and certain noncontinuous functions, Note Mat. 10 (1990), no. 1, 37-45.

[9] N. Levine, A decomposition of continuity in topological spaces, Amer. Math. Monthly 68 (1961), 44-46.

[10] _ Semi-open sets and semi-continuity in topological spaces, Amer. Math. Monthly 70 (1963), 36-41.

[11] S. N. Maheshwari and S. S. Thakur, On $\alpha$-irresolute mappings, Tamkang J. Math. 11 (1981), no. 2, 209-214.

[12] A. S. Mashhour, M. E. Abd El-Monsef, and S. N. El-Deeb, On precontinuous and weak precontinuous mappings, Proc. Math. Phys. Soc. Egypt (1982), no. 53, 47-53.

[13] A. S. Mashhour, I. A. Hasanein, and S. N. El-Deeb, $\alpha$-continuous and $\alpha$-open mappings, Acta Math. Hungar. 41 (1983), no. 3-4, 213-218.

[14] B. M. Munshi and D. S. Bassan, Super-continuous mappings, Indian J. Pure Appl. Math. 13 (1982), no. 2, 229-236.

[15] O. Njȧstad, On some classes of nearly open sets, Pacific J. Math. 15 (1965), 961-970.

[16] T. Noiri, On $\delta$-continuous functions, J. Korean Math. Soc. 16 (1980), no. 2, 161-166.

[17] T. Noiri and B. Ahmad, On semi-weakly continuous mappings, Kyungpook Math. J. 25 (1985), no. 2, 123-126.

[18] T. Noiri and S. M. Kang, On almost strongly $\theta$-continuous functions, Indian J. Pure Appl. Math. 15 (1984), no. 1, 1-8.

[19] M. K. Singal and A. R. Singal, Almost-continuous mappings, Yokohama Math. J. 16 (1968), 63-73.

MAHIDE KÜÇÜK: DePARTMENT OF MATHEMATICS, ANADOlu UNIVERSiTY, ESKiŞEHIR, TURKEY E-mail address: mkucuk@anadolu.edu.tr

YALÇIN KÜÇÜK: DEPARTMENT OF MATHEMATICS, ANADOLU UNIVERSITY, ESKIŞEHIR, TURKEY

E-mail address: ykucuk@anadolu.edu.tr

URL: http: //www. anadolu. edu.tr 


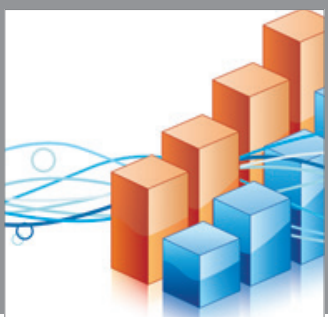

Advances in

Operations Research

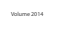

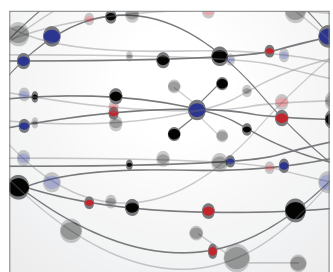

\section{The Scientific} World Journal
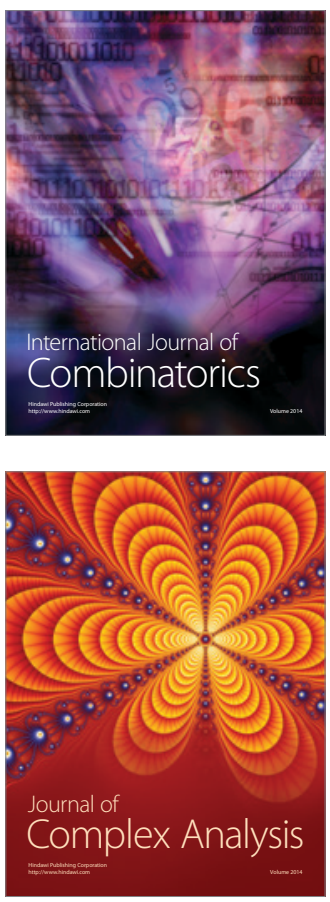

International Journal of

Mathematics and

Mathematical

Sciences
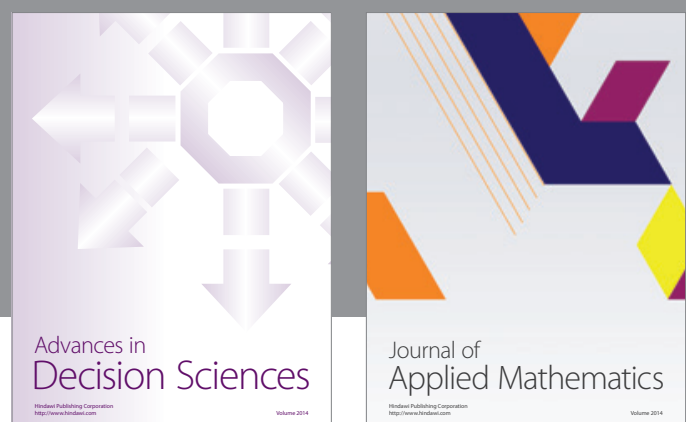

Journal of

Applied Mathematics
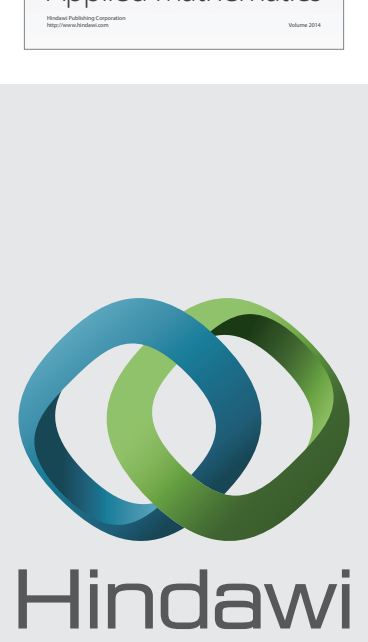

Submit your manuscripts at http://www.hindawi.com
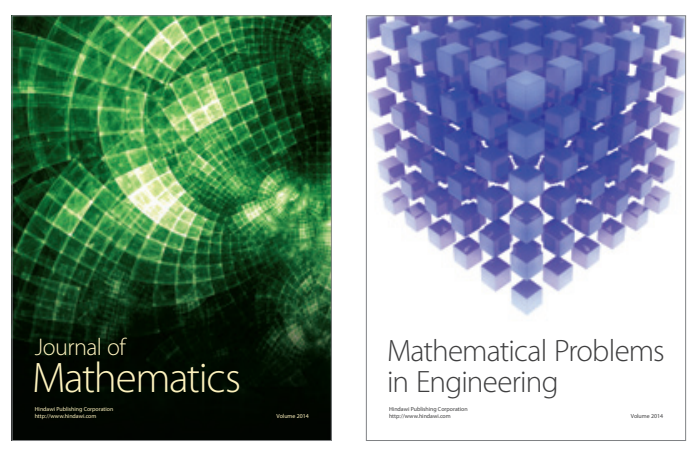

Mathematical Problems in Engineering
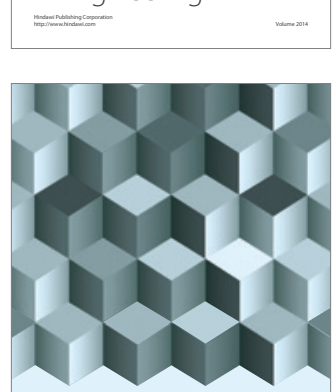

Journal of

Function Spaces
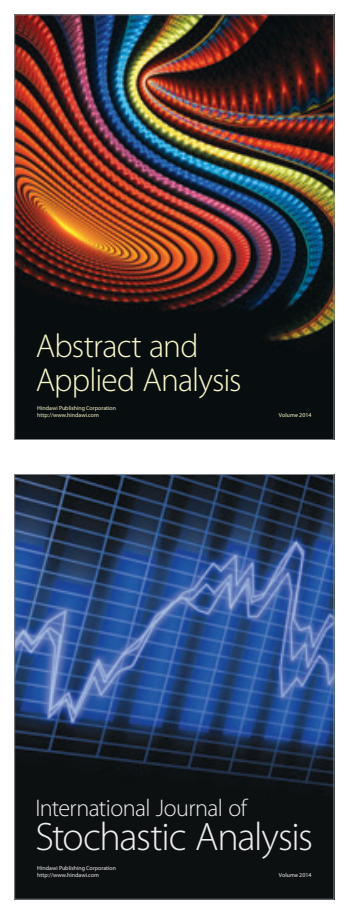

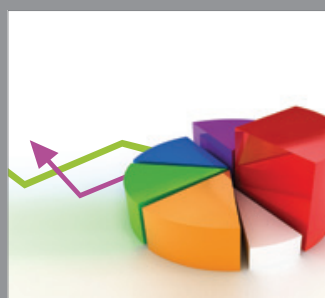

ournal of

Probability and Statistics

Promensencen
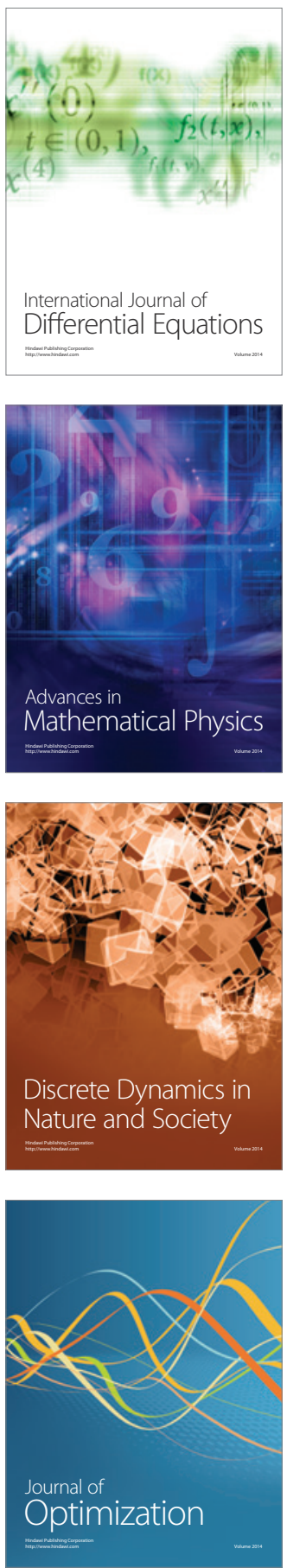